\title{
New MAPS for misfolded proteins
}

Norbert Volkmar*, Emma Fenech* and John C Christianson

*these authors contributed equally to this work

Ludwig Institute for Cancer Research, University of Oxford, Oxford OX2 6TT, United Kingdom

Clearing misfolded proteins from the cytoplasm is essential to maintain cellular homeostasis. Now a parallel clearance system is described that uses the deubiquitinase USP19 to enable secretion of misfolded cytoplasmic proteins when conventional proteasomal degradation is compromised. Misfolding-Associated Protein Secretion (MAPS) has important implications for protein quality control and prion-like transmission

Eukaryotic cells have evolved a diverse range of protein quality control (PQC) mechanisms to prevent, respond to and resolve proteotoxic stress elicited by proteins misfolding and accumulating in the cytoplasm ${ }^{1}$. Aberrant proteins may aggregate, undergo sequestration, be remediated by chaperone-assisted refolding, or be degraded by pathways including the ubiquitin-proteasome system, autophagy, and breakdown by lysosomes. Proteins misfolding in the cytoplasm may also be released into the extracellular environment independently of conventional secretion via the ER-Golgi network, notably in the cases of aggregation-prone $\alpha$-synuclein and tau proteins ${ }^{2}$, but the mechanisms remain under debate. It has been proposed that forms lacking canonical endoplasmic-reticulum (ER) targeting signals are subject to unconventional protein secretion (UPS) through mechanisms that include packaging of cytoplasmic cargo into exosomes ${ }^{3}$, autophagosome-like compartments or secretory lysosomes ${ }^{4}$, which then fuse with the plasma membrane to deposit their cargo extracellularly. One point of contention has been that these mechanisms do not differentiate between folded and misfolded client proteins, and a process specifically targeting aberrant cytosolic proteins had not been described. In this 
issue, Lee et $a l^{5}$ appear to resolve this dilemma by describing a mechanism capable of selectively recognising, packaging and secreting misfolded cytoplasmic proteins from the cell when proteasome activity is impaired or overburdened.

Of the over 100 deubiquitinases (dUb) expressed in the mammalian proteome, Ubiquitin-specific Protease 19 (USP19) is the only one anchored in the ER membrane and has been shown previously to be involved in $\mathrm{PQC}^{6}$. While investigating a role for USP19 in conventional secretion, Lee et al instead observed increased secretion of their control protein GFP with USP19 overexpression. Not attributable to disruption of plasma membrane integrity, this observation was even more apparent with model substrates misfolded in the cytoplasm, including a poorly folded GFP truncation (GFP1-10), unassembled Ubl4A, and mutant a-synuclein forms associated with Parkinson's disease. Release of these misfolded cytoplasmic forms into the extracellular space required both the catalytic deubiquitinating activity and ER-membrane localisation of USP19. Overexpressing USP19 increased secretion of misfolded cytoplasmic substrates whereas depleting it through siRNA knockdown or CRISPR knockout, strongly impaired release of cargo from cells. USP19 also showed a preference for misfolded or unassembled proteins. In vitro assays demonstrating preferential recognition of denatured luciferase by USP19 also suggested that the dUb contains a "chaperone-like" activity capable of general misfolded protein recognition. This previously unidentified USP19 activity requires its USP domain and is importantly, independent of Hsp90, a cytosolic chaperone known to associate with USP197 which could have provided such activity. These observations collectively pointed towards a new PQC process (Figure 1) based on unconventional secretion, for which the authors coined the term MAPS (MisfoldingAssociated Protein $\underline{\text { Secretion}) . ~}$

MAPS appears to be distinct from other known unconventional secretion pathways that are dependent on exosomes ${ }^{5}$, secretory lysosomes or autophagosomes ${ }^{4}$, instead using USP19 for both cargo recognition and subsequent packaging for export. USP19 overexpression promoted secretion of mutant $\alpha$-synuclein but not the exosome-dependent tau protein, indicating different strategies for proteotoxin 
secretion are present and may exert some not currently understood selectivity . While investigating the functional interplay between MAPS and proteasome mediated PQC, misfolded substrates were observed to accumulate with proteasome inhibition and increasingly secreted. Malfolded, aggregation-prone proteins would typically be polyubiquitinated and efficiently targeted for degradation by proteasomes. However these findings point towards MAPS as a PQC pathway for misfolded proteins operating in parallel to the conventional system and called into action when proteasome activity becomes compromised. Interestingly, the observed impaired secretion by active-site cysteine mutants of USP19 and failure to detect ubiquitinated forms of secreted cargo suggest that removal of polyubiquitin chains from candidate cargo is a pre-requisite for MAPS. USP19 CRISPR knockout cells exposed to proteasome inhibitor exhibited higher levels of cell death, indicating that disrupting both the primary and secondary clearance routes for cytotoxic proteins is not tolerated.

Subcellular fractionation and confocal microscopy revealed that the misfolded cargo for MAPS localise to the ER membrane where their association correlate with secretion efficiency. Following recruitment to USP19 at the ER membrane, MAPS cargos are encapsulated into vesicles that subsequently fuse with the plasma membrane.As other UPS pathways use distinct membrane-bounded vesicles ${ }^{8}$, the authors screened a range of markers and found that MAPS vesicles are positive for Rab9, indicating their identity as late endosomes (LE) ${ }^{9}$ and further emphasising MAPS as a pathway distinct from other forms of release. Super-resolution microscopy showed that MAPS cargoes are in the lumen (or limiting membranes) of the Rab9 positive LEs. How USP19 transfers cargo to LEs for MAPS remains unclear. These encapsulating LEs are not targeted to lysosomes for degradation, but rather translocate to the plasma membrane where they fuse to release misfolded cargo from their lumen into the extracellular space. Cargo-containing MAPS vesicles also contain the fusion-machinery proteins VAMP7 and VAMP8, members of the SNARE family that mediate fusion of membrane compartments ${ }^{10,11}$. Overexpression of either VAMP7 or VAMP8 result in the increased export of the MAPS cargo GFP110 , implying fusion of MAPS vesicles with the plasma membrane as the mechanism 
of cargo release. Thus, MAPS appears to be a unique form of UPS that uses LEs as carriers for its potentially proteotoxic cargo.

The fate of secreted MAPS cargo and implications for organismal viability is still unknown. Whereas malfolded forms could undergo proteolysis or be internalised and degraded by neighbouring cells, proteotoxic cargo jettisoned by MAPS could be a source of extracellular deposits responsible for cell-to-cell transmission that hallmark neurodegenerative disorders such as Huntington's disease ${ }^{12}$ and $\alpha$ synucleinopathies $^{13}$. The identification of disease-associated $\alpha$-synuclein variants as clients of MAPS holds particular promise for understanding the general mechanisms of PQC in neurodegenerative disorders. As the active site of USP19 is required for MAPS, this deubiquitinating enzyme could be an attractive target for small molecule inhibitors. Modulation of MAPS though USP19 inhibition might therefore be a means of controlling the spread of disease-related, toxic misfolded protein species. It should be noted however that inhibiting MAPS may cause misfolded proteins to be retained intracellularly, potentially exacerbating existing stress levels and accelerating cell death and their undesirable release into the extracellular milieu, where they pose a similar risk to surrounding cells ${ }^{13}$. Other features of MAPS such as the chaperone activity of USP19 or steps encapsulating cargoes by LEs may prove equally suitable for disrupting the adaptive capacity of MAPS and further study is clearly required.

The mechanisms used by eukaryotic cells to maintain and restore protein homeostasis have increasingly been receiving attention a viable points for therapeutic intervention. Identifying the MAPS pathway as another avenue of protein clearance, constitutively active but increasingly engaged when proteasome activity is compromised, is important to fully appreciate how these adaptive mechanisms allow cells to maintain homeostasis and remain viable. It will be essential to understand the contribution MAPS makes to protein homeostasis under both physiological and pathological conditions, as there may be implications for therapeutic strategies to a range of diseases. For example, the capability of MAPS to clear misfolded proteins may be particularly relevant for strategies involving proteasome inhibitors as therapeutic agents. Proteasome inhibitors have proved effective in the treatment of select haematological malignancies ${ }^{14}$. As with autophagy, USP19-dependent MAPS 
may complementarily enable cellular adaptation to the loss of proteolytic activity, thus avoiding a fate of stress-induced apoptosis. Consequently, further inquiry into understanding how MAPS may be manipulated, might help to overcome the eventual resistance observed with proteasome inhibitor-based therapeutic strategies. The description of MAPS as an auxiliary mechanism to react to and abate proteotoxic stress is an exciting first step in elucidating the involvement of this pathway in both physiological and pathological processes.

The authors declare no competing financial interests

\section{REFERENCES}

1. Wolff, S., Weissman, J. S. \& Dillin, A. Cell 157, 52-64 (2014).

2. Oueslati, A., Ximerakis, M. \& Vekrellis, K. Exp Neurobiol 23, 324-336 (2014).

3. Saman, S. et al. Journal of Biological Chemistry 287, 3842-3849 (2012).

4. Nickel, W. Curr. Opin. Biotechnol. 21, 621-626 (2010).

5. Lee, J.-G., Takahama, S., Zhang, G., Tomarev, S. I. \& Ye, Y. Nat Cell Biol XX, XX (2016).

6. Hassink, G. C. et al. EMBO Rep 10, 755-761 (2009).

7. Lee, J.-G., Kim, W., Gygi, S. \& Ye, Y. J Biol Chem 289, 3510-3517 (2014).

8. Zhang, M. \& Schekman, R. Cell biology. Science 340, 559-561 (2013).

9. $\quad$ Soldati, T., Rancaño, C., Geissler, H. \& Pfeffer, S. R. J Biol Chem 270, 2554125548 (1995).

10. Advani, R. J. et al. The Journal of Cell Biology 146, 765-776 (1999).

11. Antonin, W., Holroyd, C., Tikkanen, R., Höning, S. \& Jahn, R. Mol Biol Cell 11, 3289-3298 (2000).

12. Ciechanover, A. \& Kwon, Y. T. Exp. Mol. Med. 47, e147 (2015).

13. Lee, H.-J., Bae, E.-J. \& Lee, S.-J. Nat Rev Neurol 10, 92-98 (2014).

14. Bross, P. F. et al. Clin. Cancer Res. 10, 3954-3964 (2004). 